\title{
Preparation, Structure and Fluorescence properties of Novel Zinc(II) Complex Containing taurine Schiff Base Ligand
}

\author{
Liyan You ${ }^{1, a}$, Yue Wang ${ }^{1, b}$ \\ ${ }^{1}$ School of Arts and Science, Jilin Agricultural Science and Technology University, Jilin 132101, \\ China
}

ayly5466@163.com, b2668975403@qq.com

Keywords: IsoIsovanillin Schiff base; complex; crystal structure; infrared analysis Abstract: In recent years, with Schiff base being applied and researched deeply in biology, IsoIsovanillinSchiff base ligands' synthesis and the coordinathion properties have become a hot spot in the research of Schiff bases compounds. This paper expounds that we keep Isovanillin and taurine as the raw material to design and synthesize Schiff base ligands, and Zinc(II) of metal compounds to form the corresponding $\mathrm{Zn}$ (II) complexes by solvent. The crystal structure of the Zinc(II) complex has been determined and studied by X-ray diffraction.

\section{Introduction}

Taurine has important physiological functions. It can promote brain tissue, intelligence and retina development, promote immune function and lipid digestion and absorption, protect myocardium and antioxidation in infants. Because of its unique pharmacological and physiological functions, it has been widely used in food, medicine and chemical industry. A lot of taurine complexes and the Taurine Schiff base metal complexes research reports, which formed at the same time sulfonate and the corresponding metal ions involved in hydrogen bonding, or just to participate in the formation of hydrogen bonds. A new type of complex $\left[\mathrm{Zn}(\mathrm{TISB})(\mathrm{phen})_{2}\right]$ was prepared by aqueous solution using TISB and phen as mixed ligands. Its structure is characterized by IR and X-ray single crystal diffraction.

\section{Experimental}

\section{Materials and physical measurements}

All solvents and chemicals purchased from commercial sources were of reagent grade and used without further purification. IR spectra were performed on a Shimizu spectrum FT-IR spectrometer with $\mathrm{KBr}$ pallets in the range of $4000 \sim 400 \mathrm{~cm}^{-1}$. The elemental analyses for $\mathrm{C}, \mathrm{H}$, $\mathrm{N}$ and $\mathrm{S}$ were carried out on a Leeman elemental analyzer. The crystal data were collected on a Rigaku Xtalab area detector diffractometer at room temperature.

\section{Synthesis of the ligand(L)}

In the reaction flask, anhydrous methanol $80 \mathrm{~mL}$, taurine $2.5 \mathrm{~g}(20 \mathrm{mmol})$ and $\mathrm{KOH} 0.112 \mathrm{~g}(20$ mmol) were added successively to dissolve them. A slow trickle of Isovanillin $3.04 \mathrm{~g} \mathrm{(20} \mathrm{mmol)} \mathrm{of}$ anhydrous methanol $(20 \mathrm{~mL})$ solution, drop after icewater cooling reaction under $4 \mathrm{~h}$. The filter cake was placed in a stationary position and filtered. The cake was washed with anhydrous methanol $(3 \times 20 \mathrm{~mL})$ and ether $(3 \times 20 \mathrm{~mL})$ in a row. The vacuum dried yellow powder, TISB $4.22 \mathrm{~g}$, was obtained with a yield of $71 \%$. IRv:1636(C=N), 1510, 1466, $1438(\mathrm{C}=\mathrm{C}), 1251(\mathrm{C}-\mathrm{O})$, 1171, 1045, 603, 535( S-O, S=O) $\mathrm{cm}^{-1}$; Anal. calcd for C10H12 NO5 SK: C 40.36, H4.03, 
$\mathrm{N} 4.69, \mathrm{~S} 12.11$; found C 40.32, H 3.91, N 4.86, S 11.95 。

\section{Synthesis of complex 1}

In the reaction bottle, methanol $(10 \mathrm{~mL})$, water $(20 \mathrm{~mL}), \mathrm{ZnCl}_{2}(0.183 \mathrm{~g}, 1 \mathrm{mmol})$ and TISB $0.148 \mathrm{~g}(0.5 \mathrm{mmol})$ are added successively to be dissolved. The reaction is about $2 \mathrm{~h}$ at normal temperature. Slowly add phen $(0.198 \mathrm{~g}, 1 \mathrm{mmol})$ and DMF a few drops, drop, reflux reaction $\left(60{ }^{\circ} \mathrm{C}\right), 5 \mathrm{~h}$. Cool to room temperature, filter, filtrate at room temperature, slowly volatile, a few days later, brown black crystal of complex 1. IR v: 3405(O-H), 1613(C = N), 1518, 1464, 1430 $(\mathrm{C}=\mathrm{C}), 1210(\mathrm{C}-\mathrm{O}), 1159,1042,619,527(\mathrm{~S}-\mathrm{O} ， \mathrm{~S}=\mathrm{O}) \mathrm{cm}^{-1}$; Anal. calcd for C34 H37 N5O10 SZn: C 53.25, H 4.81, N 9.15, S 4.20; found C 53.06, H 5.08, N 9.35, S 9.68.

\section{Structure determination}

A suitable single crystal of complex1 $(0.36 \mathrm{~mm} \times 0.24 \mathrm{~mm} \times 0.11 \mathrm{~mm})$ was selected and glued on the tip of a glass fiber. The crystal structure was determined at $298 \mathrm{~K}$ on an X-ray diffractometer, a Rigaku Xtalab detector diffractometer by using a $\mathrm{CuK \alpha}$ radiation $(\lambda=0.154 \AA)$. All structures were solved using direct methods with SHELXS and refined by full-matrix leastsquares on $F 2$ using the SHELXL14 software within OLEX2. All the non-hydrogen atoms were refined anisotropically with displacement parameters. The hydrogen atoms were generated geometrically and treated by a mixture of independent and constrained refinement using a riding model. Crystal data and structure parameters for the title complex are listed in Table 1.

Table 1 Crystal data for Zinc(II) Compound

\begin{tabular}{ll}
\hline Polymers & Complex1 \\
\hline Formula & C34 H37 N5 O10 SZn \\
Formula weight & 772.63 \\
Temperature(K) & 298 \\
Wavelength(A) & 0.154 \\
Crystal sizes(mm) & $0.16 \mathrm{~mm} \times 0.24 \mathrm{~mm} \times 0.17 \mathrm{~mm}$ \\
Color & colorless \\
Crystal system & monoclinic \\
Spaces group & $\mathrm{C} 2 / \mathrm{c}$ \\
$\mathrm{a}(\mathrm{A})$ & $20.254(1)$ \\
$\mathrm{b}(\mathrm{A})$ & $16.128(1)$ \\
$\mathrm{c}(\mathrm{A})$ & $12.657(3)$ \\
$\alpha\left(^{\circ}\right)$ & $102.561(4)$ \\
$\beta\left(^{\circ}\right)$ & $98.372(5)$ \\
$\gamma\left({ }^{\circ}\right)$ & $118.244(2)$ \\
$\mathrm{Z}$ & 4 \\
\hline
\end{tabular}

Table 2 elected Bonds Lengths (nm) and Bonds Angles( ${ }^{\circ}$ )

\begin{tabular}{llll|ll}
\hline \multicolumn{2}{c|}{ Bond Lengths } & \multicolumn{4}{c}{ Bond Angles } \\
\hline Zn1-O1 & $1.862(1)$ & O1-Zn1-N2 & $90.92(1)$ & O1-Zn1-N4 & $83.42(5)$ \\
Zn1-N2 & $1.959(4)$ & O1-Zn1-N3 & $171.69(5)$ & N3-Zn1-N4 & $91.79(5)$ \\
Zn1-N5 & $1.891(7)$ & O1-Zn1-N1 & $90.64(2)$ & N2-Zn1-N5 & $174.82(1)$ \\
Zn1-N3 & $1.986(3)$ & N3-Zn1-N1 & $94.05(6)$ & N5-Zn1-N3 & $94.21(1)$ \\
Zn1-N1 & $1.994(6)$ & N5-Zn1-N4 & $82.13(2)$ & N5-Zn1-N1 & $92.64(3)$ \\
Zn1-N4 & $1.897(5)$ & O1-Zn1-N5 & $83.74(2)$ & N2-Zn1-N4 & $92.37(1)$ \\
& & N2-Zn1-N3 & $81.21(8)$ & N1-Zn1-N4 & $175.25(7)$ \\
& & N2-Zn1-N1 & $83.77(4)$ & & \\
\hline
\end{tabular}

Symmetry transformations used to generate equivalent atoms: $\# 1: \mathrm{x}, \mathrm{y}, \mathrm{z}$ 


\section{Results and Discussion}

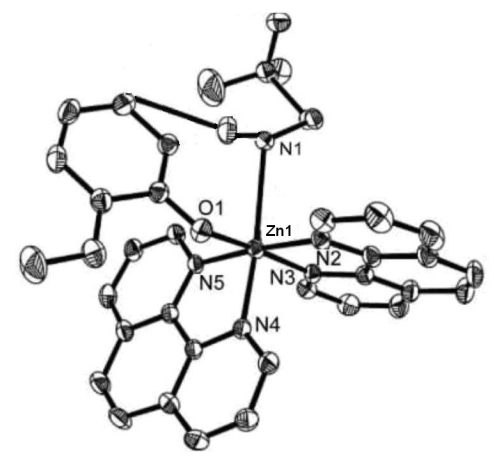

Fig.1 Crystal Structure of complex 1

The center Zn (II) of complexes 1 with two phen molecules, respectively, coordinates with four $\mathrm{N}$ atoms. $\mathrm{A} \mathrm{N}$ atom on the $\mathrm{C}=\mathrm{N}$ double bond on the TISB, the $\mathrm{O}$ atoms of deprotonated phenolic hydroxyl coordinate to form six coordinated deformation of the octahedron. Among them, N (1) and $\mathrm{N}(4)$ are in the axial position, $\mathrm{N}(2), \mathrm{N}(3), \mathrm{N}(5)$ and $\mathrm{O}(1)$ are in the equatorial plane.

The bond angles formed with the central $\mathrm{N}(2)-\mathrm{Zn} 1-\mathrm{N}(3)$ is 81.21 (8) degrees. The bond angles formed with the central N (3) - Zn1-N (5) is equal to 94.21 (1) degrees. The bond angles formed with the central N (5) - Zn1-O (1) is about 83.74. (2) degrees. The bond angles formed with the central O (1) - Zn1- N (2) is 90.92 (1). The sum of the key angles is close to 360 degrees (350.08 degree), and the center Zinc (II) is separated from the plane N (2), /N (3), /N (5), /O (1) to $0.127 \mathrm{~nm}$. From the bond length data, it is known that the $\mathrm{Zn} 1-\mathrm{N}$ which is in the moleculer of phen, bond grows between 1.862 and 1.994, and is similar to other Zinc phenanthroline complexes. The determination of the dihedral angle between the two phen molecular plane is 81.93 degrees.

Among the complexes 1 , there are two kinds of $\pi-\pi$ stacking action between the rings of phen and the benzene ring of the TISB ligand, and the corresponding distances of their $\pi$ - $\pi$ stacking are 3.5167 and 3.5476 respectively. Although the sulfonate in the TISB ligand does not participate in the coordination of metal ions, it stabilizes complexes 1, it is through these two kinds of PI stacking action and hydrogen bonding to form a three-dimensional structure. Complexes 1 of the structure by hydrogen bonds formed with water molecules. In complexes 1, it is through these two kinds of PI stacking action and hydrogen bonding to form a three-dimensional structure.

\section{IR Analysis}

The infrared spectra of ligands and coordination polymers were determined by the potassium bromide tabletting method in the range of $4000-400 \mathrm{~cm}^{-1}$ wavenumbers. There are strong peaks which were found near the $1613 \mathrm{~cm}^{-1}$. It indicates the formation of $\mathrm{C}=\mathrm{N}$ Schiff base. The absorption peaks of $162,1038,624$ and $524 \mathrm{~cm}^{-1}$ are characteristic absorption peaks of sulfonic group. We also find absorption peaks at $1210 \mathrm{~cm}^{-1}$ which belong to $\mathrm{C}-\mathrm{O}$ of phenol oxygen. The absorption peak at 1518, 1464, $1430 \mathrm{~cm}^{-1}$ belongs to the benzene. It is obviously that -OH stretching frequency of water molecules coordinate because of the presence of the broad band around $3405 \mathrm{~cm}^{-1}$. Comparison with the infrared spectra of the ligand and the synthesized Zinc complex, it makes clear that the coordination bonds have formed.

\section{Thermal Stability Behavior}

The thermal decomposition curves of the complexes were measured at temperatures of $25-450^{\circ} \mathrm{C}$. The thermal decomposition of the complex is divided into two parts. The first weightlessness process ranges from $25-250^{\circ} \mathrm{C}$ to $0.598 \%$, and the weight loss rate of the complexes is lost. The weight loss rate of the complexes between second weight loss processes at $250-450{ }^{\circ} \mathrm{C}$ is $29.288 \%$, which is the decomposition of Schiff base complexes. The final residual rate was 
$70.114 \%$ for $\mathrm{Zn}-\mathrm{O}$ and some residual groups for Schiff base

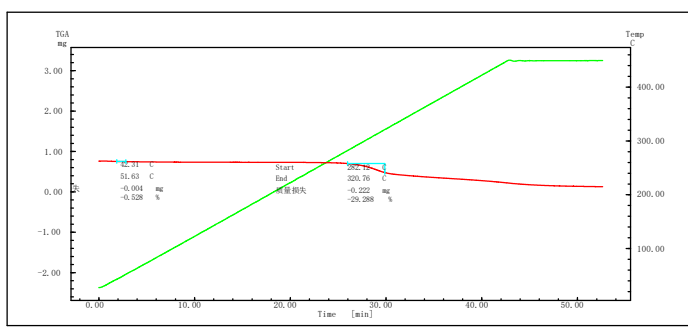

Fig.2 The thermogravimetric analysis of the complex 1

\section{Fluorescence properties}

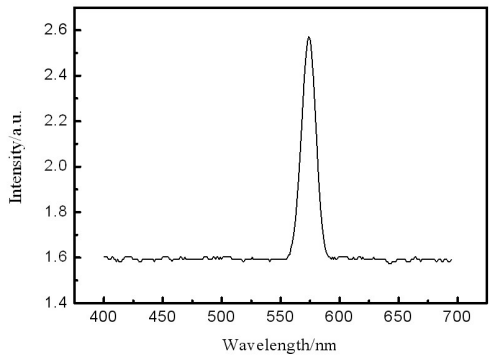

Fig.3 Fluorescence properties of complex1(Emission:575nm)

The fluorescence luminescence photometer was used to display the spectral range of $400 \mathrm{~nm} \sim 700 \mathrm{~nm}$ The fluorescence spectra of the complex 1 are shown in Fig. 3. As shown as in Fig. 3, the emission wavelength of the complex 1 is about at $575 \mathrm{~nm}$. As we can see from Fig. 3, the wave crest has a pronounced blue shift (moving about $5 \mathrm{~nm}$ to the left), indicating that the ligand affects the emission wavelength of $\mathrm{Zn}^{2+}$. In addition, the height of the peak is higher than the fluorescence intensity of $\mathrm{Zn}^{2+}$ according to the relevant literature, indicating that the ligand sensitized the fluorescence of metal ions.

\section{Conclusions}

In this paper, we have presented the Zinc(II) complex [Zn(TISB)(phen) $)_{2}$ (TISB $=$ the Schiff base synthesized by Isovanillin and taurine). The crystal structure of the Zinc (II) complex has been determined and studied by X-ray diffraction, which is also characterized by elemental analysis, IR spectra, thermogravimetric analysis. Fluorescence properties also have been studied in order to investigate the thermol behavior carefully.

\section{Acknowledgements}

This work was supported by Jilin college students innovation and entrepreneurship training program(2017013).

\section{References}

[1] Y. Kato, M. Furutachi, Z. Chen, H. Mitsunuma, S. Matsunaga. A Homodinuclear Mn(III) 2-Schiff Base Complex for Catalytic Asymmetric 1,4-Additions of Oxindoles to Nitroalkenes.

J. Am. Chem. Soc.. 2009, 131 (26): p9168-9169.

[2] Yan B, Kong L, Zhou B. A luminescent covalently bonded rare earth hybrid material by functionalized trifluoroacetylacetone linkage[J]. Journal of Non-Crystalline Solids. 2009, 355(22-23): p1281-1284.

[3] Xuzhong Luo, Xuefen Wang, Sanxie Wu, et al.. Strong blue-fluorescence-emitted stable 
monolayers formed in organic solvents by a coordination polymer with long-chained bis-Schiff base. Journal of Colloid and Interface Science, 2003, 258: p432-434.

[4] A.H. Kianfar , A.K.M. Wan , M. Dinari , M.H. Azarian , F.Z. Khafri. Novel nanohybrids of cobalt(III) Schiff base complexes and clay: Synthesis and structural determinations. Spectrochimica Acta Part A: Molecular and Biomolecular Spectroscopy. 2014, 127(1644): p422-428

[5]G. M. Sheldrick, SHELX-97, Program for the solution and Refinement of Crystal Structures. University of Gottingen: Gottingen, Germany, (1997). 